\title{
Role of the RAGE Axis during the Immune Response after Severe Trauma: A Prospective Pilot Study
}

\author{
Florian Uhle, ${ }^{1}$ Christoph Lichtenstern, ${ }^{1}$ Thorsten Brenner, ${ }^{1}$ Thomas Fleming, \\ Christian Koch, ${ }^{3}$ Andreas Hecker, ${ }^{4}$ Christian Heiss, ${ }^{5}$ Peter Paul Nawroth, ${ }^{2}$ Stefan Hofer, ${ }^{1}$ \\ Markus Alexander Weigand, ${ }^{1}$ and Katja Weismüller ${ }^{3}$ \\ ${ }^{1}$ Department of Anesthesiology, Heidelberg University Hospital, 69120 Heidelberg, Germany \\ ${ }^{2}$ Department of Medicine I and Clinical Chemistry, Heidelberg University Hospital, 69120 Heidelberg, Germany \\ ${ }^{3}$ Department of Anaesthesiology and Intensive Care Medicine, Justus-Liebig-University, 35392 Giessen, Germany \\ ${ }^{4}$ Department of General and Thoracic Surgery, Justus-Liebig-University, 35392 Giessen, Germany \\ ${ }^{5}$ Department of Trauma, Hand and Reconstructive Surgery, University Hospital of Giessen-Marburg GmbH, Campus Giessen, \\ 35392 Giessen, Germany
}

Correspondence should be addressed to Florian Uhle; florian.uhle@med.uni-heidelberg.de

Received 6 October 2015; Revised 24 November 2015; Accepted 9 December 2015

Academic Editor: Marc Pouliot

Copyright (C) 2015 Florian Uhle et al. This is an open access article distributed under the Creative Commons Attribution License, which permits unrestricted use, distribution, and reproduction in any medium, provided the original work is properly cited.

\begin{abstract}
Background. Severe traumatization induces a complex pathophysiology, driven by the patient's own immune system. The initial activation is a result of damage-associated molecular patterns, which are released from disrupted and dying cells and recognized by immune receptors, for example, RAGE. In this study we aimed to evaluate the contribution of the RAGE axis to early and late immune responses. Methods. We enrolled 16 patients with severe trauma together with 10 patients after major abdominal surgery and 10 healthy volunteers. Blood samples were taken on admission and every $48 \mathrm{~h}$ for a total of 8 days. Plasma concentrations of various RAGE ligands as well as RAGE isoforms and IL-6 were measured by ELISA. Monocyte surface expression of RAGE and HLA-DR was assessed by flow cytometry. Results. High and transient levels of IL-6 and methylglyoxal characterize the early immune response after trauma, whereas samples from later time points provide evidence for a secondary release of RAGE ligands. Conclusion. Our results provide evidence for a persisting activation of the RAGE axis while classical mediators like IL-6 disappear early. Considering the immunocompromised phenotype of the monocytes, the RAGE ligands might be substantial contributors to the well-known secondary stage of impaired immune responsiveness in trauma patients.
\end{abstract}

\section{Introduction}

The treatment of traumatic injuries is a basic everyday task of the healthcare systems around the world. The situation becomes drastically more challenging when the severity and combination of injuries result in a life-threatening condition for the patient. These severe traumatic injuries cause globally more than 5 million deaths per year and this number might further increase over the next years $[1,2]$. Besides the actual fatality rate, the treatment, remaining disabilities, and bodily impairments represent a high individual as well as socioeconomic burden [3]. Severe injuries originate from a variety of causes, not least from armed conflicts, but in the absence of war, traffic accidents are the leading cause of hospitalization in developed as well as third-world countries. As a consequence of the injuries, the patient's homeostasis collapses and a highly complex pathophysiological network is triggered [4]. Main drivers at it are both interwoven systems of coagulation and inflammation [5], the latter one initially activated by immunogenic damage-associated molecular patterns (DAMPs) released from disrupted and necrotic cells [6]. Due to the large amount of injuries and released mediators, trauma patients often present with a systemic inflammatory response syndrome (SIRS) at first. This is a result of DAMPs activating immune cells by binding to their corresponding pattern recognition receptor, for example, the Receptor for Advanced Glycation End Products (RAGE). RAGE is a multiligand immune receptor that recognizes 
different DAMPs, including proteinergic ligands like highmobility group box 1 protein (HMGB1) or S100 proteins as well as metabolically modified proteins and lipids, known as Advanced Glycation End Products (AGEs) [7-9]. As a consequence of RAGE binding, proinflammatory signaling cascades are activated, ultimately leading to the activation of $\mathrm{NF}-\kappa \mathrm{B}$ and changes in target gene expression. AGEs thereby arise from a nonenzymatically reaction (Maillard reaction) in conditions of high oxidative stress in combination with the availability of glucose [10]. Several conditions, for example, diabetes and its complications like atherosclerosis, have been shown to be causally driven by these compounds and ligands [11] and soluble forms of RAGE have been identified and characterized in many others, including acute systemic inflammatory conditions like sepsis [12-14]. Two prominent soluble isoforms with distinct mechanisms of generation are known, endogenous secretory RAGE (esRAGE) and soluble RAGE (sRAGE). While esRAGE is the consequence of an alternative splicing event followed by secretion, sRAGE derives from the proteolytic cleavage of the membranebound receptor. After an initial activation, the organism tries to balance the immune reaction, leading to a compensatory phase of immune dysfunction in trauma patients [15]. This later phase is of high relevance to the patients, as they are rendered highly vulnerable for infections during this time of recovery, not only because of their immune status, but also due to the disruption of physical body barriers like, for example, skin or lung epithelium.

Following our earlier own results and results from other groups [16, 17], we wanted to extend our knowledge about the participation of RAGE and its ligands in the systemic pro- and anti-inflammatory immune response after trauma. In order to achieve this, we conducted a prospective observational cohort study and measured plasmatic concentrations of known ligands as well as soluble and membrane-bound receptor isoforms. Surprisingly, we are able to show different spatiotemporal patterns for the ligands of RAGE, hinting at a persistent basal "RAGEing" milieu, which might contribute to the immune dysfunction of the patients.

\section{Patients and Methods}

2.1. Patients. This observational clinical study was conducted in the Surgical Intensive Care Unit of the University Hospital Giessen, Germany, after approval by the local ethics committee (Ethics Committee of the Medical Faculty of the Justus Liebig University Giessen: Trial code 127/09/German Clinical Trials Register: DRKS00000480). Informed consent was obtained from all patients and volunteers enrolled. In case of a patient not being able to give consent, the legal representative was informed in advance. Overall, 16 patients after severe trauma as characterized by an Injury Severity Score (ISS) $\geq$ 16 were enrolled in this study. In addition, 10 patients after major abdominal surgery (Table 1 ) and 10 healthy volunteers were included. For all groups, the fulfillment of any of the following criteria led to an exclusion from the study: age $<18$ years, terminal renal insufficiency, diabetes mellitus (types I and II), autoimmune disorder (e.g., Morbus Crohn,
TABLE 1: Baseline characteristics of the three investigated groups.

\begin{tabular}{|c|c|c|c|}
\hline & Trauma & Surgical & Healthy \\
\hline Patients & $16(100)$ & $10(100)$ & $10(100)$ \\
\hline Sex, male & $14(87.5)$ & $8(80)$ & $6(60)$ \\
\hline Age, y & $44.1(19-69)$ & $63(48-76)$ & $38(28-55)$ \\
\hline 28-day mortality & $2(11)$ & & \\
\hline ISS & $34.1(19-51)$ & & \\
\hline \multicolumn{4}{|l|}{ Cause of injury } \\
\hline Car accident & $7(43.75)$ & & \\
\hline Motorcycle accident & $3(18.75)$ & & \\
\hline Pedestrian accident & $1(6.25)$ & & \\
\hline Fall & $2(12.5)$ & & \\
\hline Unknown or other & $3(18.75)$ & & \\
\hline \multicolumn{4}{|l|}{ Injuries $^{\#}$} \\
\hline $\begin{array}{l}\text { Fracture of the } \\
\text { extremities }\end{array}$ & $9(56.25)$ & & \\
\hline $\begin{array}{l}\text { Fracture of the axial } \\
\text { skeleton }\end{array}$ & $10(62.5)$ & & \\
\hline Abdominal trauma & $12(75)$ & & \\
\hline Thorax trauma & $14(87.5)$ & & \\
\hline Head injury & $5(31.25)$ & & \\
\hline \multicolumn{4}{|l|}{ Surgical procedure } \\
\hline Esophagectomy & & $2(20)$ & \\
\hline Pancreatectomy & & $2(20)$ & \\
\hline Gastrectomy & & $3(30)$ & \\
\hline Hemihepatectomy & & $1(10)$ & \\
\hline Duodenectomy & & $1(10)$ & \\
\hline Hemicolectomy & & $1(10)$ & \\
\hline
\end{tabular}

Values are given either as mean (range) for age and ISS or as $n$ (\% total); ${ }^{\#}$ multiple naming possible.

rheumatoid arthritis), atherosclerosis with history of myocardial ischemia/coronary artery bypass grafting/percutaneous transluminal coronary angioplasty, and pregnancy.

Blood samples were taken from trauma patients within $24 \mathrm{~h}$ after hospital admission and subsequently every 2 days (for a maximum of 8 days), as long as the patients were present in the ICU. Blood was drawn only once in case of surgical patients (within $24 \mathrm{~h}$ after end of surgery) and healthy volunteers.

2.2. Sample Preparation and ELISA. Blood was drawn directly into anticoagulative EDTA tubes and immediately processed. Plasmatic and cellular fractions were separated by centrifugation with $1.200 \times \mathrm{g}$ for 10 minutes at room temperature. After removal, plasma was stored in aliquots at $-80^{\circ} \mathrm{C}$ until further analysis. Every sample was only thawed once before analysis.

Plasmatic factors were analyzed using commercially available ELISA according to the manufacturer's instructions: sRAGE (R\&D Systems, Minneapolis, USA), esRAGE (BBridge International, Cupertino, USA), interleukin-6 (IL-6) (R\&D Systems, Minneapolis, USA), AGE, CML, and MG 
(all the three from Cell Biolabs, Inc., San Diego, USA), S100A8 (RayBiotech, Norcross, USA), S100A12 (MBL International, Woburn, USA), and HMGB1 (Shino-Test Corporation, Tokyo, Japan).

2.3. Flow Cytometry. Expression of RAGE and HLA-DR was assessed by flow cytometry. For the quantitative measurement of HLA-DR, $50 \mu \mathrm{L}$ of whole blood was incubated with $20 \mu \mathrm{L}$ of Quantibrite HLA-DR/monocyte antibody cocktail (BD Bioscience, San Jose, USA) for 30 minutes at $4^{\circ} \mathrm{C}$ in the dark. Afterwards, erythrocytes were lysed by the addition of $450 \mu \mathrm{L}$ 1x FACS lysing solution (BD Bioscience, San Jose, USA) and further incubation for 15 minutes at room temperature. Measurements were performed on a FACSCalibur cytometer (BD Bioscience, San Jose, USA). At each time point a calibration curve was obtained by measuring beads with distinct fluorescence properties (Quantibrite PE Beads, BD Bioscience, San Jose, USA). Results were calculated according to the manufacturer's instructions and given as average number of HLA-DR molecules on each monocyte.

In case of RAGE, $100 \mu \mathrm{L}$ whole blood was incubated for 30 minutes with $0.2 \mu \mathrm{g}$ anti-CD14-FITC (category number 301804, BioLegend, San Diego, USA) together with $4 \mu \mathrm{g}$ antiRAGE-APC (category number sc-80652, Santa Cruz Biotech, Dallas, USA) or an equivalent amount of isotype control antibody (sc-2889, Santa Cruz Biotech, Dallas, USA). Erythrocyte lysis was performed by addition of $2 \mathrm{~mL}$ FACS lysing solution followed by an additional incubation (15 minutes) and two washing steps. Measured mean fluorescence intensity was compared to an APC calibration curve (Bangs Laboratories, Fishers, USA) to obtain a robust quantitative result expressed in molecules of equivalent soluble fluorochrome (MESF).

2.4. Measurement of Methylglyoxal with High-Performance Liquid Chromatography (HPLC). The concentrations of methylglyoxal (MG) in plasma were determined by derivatization with 1,2-diamino-4,5-dimethoxybenzene and HPLC of the quinoxaline adduct by fluorescence detection as described earlier $[18,19]$.

2.5. Statistics. All analysis and visualizations were performed using SPSS Statistics for Mac version 21 (IBM, Armonk, NY). In general, boxplots were used for graphical representation. For group comparisons, the nonparametrical Mann-Whitney $U$ test was used. For the comparison of more than two groups, Kruskal-Wallis test for global differences was performed initially. For analysis of correlation, nonparametric Spearman $\rho$ analysis was performed. Levels of significance are depicted as symbols within the figures with ${ }^{*} P<0.05,{ }^{* *} P<0.01$, and ${ }^{* * *} P<0.001$.

\section{Results}

3.1. Study Population. The investigated cohort of trauma patients mainly consisted of males $(87.5 \%)$ of middle age (median: 44.1 years) with a median ISS of 34.1, representing a "typical" trauma cohort with severe injuries. These mainly resulted from traffic accidents, from which car accidents as a (co)driver were the leading cause (43.8\%). As a consequence, the majority of patients presented with injuries of the thorax (87.5\%) and/or the abdomen $(75 \%)$, whereas head injuries were only present in around one-third of all patients (Table 1). In terms of routine laboratory markers, the group of trauma patients showed an early increase in CRP, while leucocytes had an overall delayed kinetic. Blood glucose levels tended to be increased over the whole observation time (Supplementary Figure 1) (see Supplementary Material available online at http://dx.doi.org/10.1155/2015/691491). The postoperative control cohort consisted of patients who were subjected to major abdominal surgery, for example, resection of the pancreas or stomach.

3.2. Soluble Isoforms of RAGE and IL-6 after Trauma. In line with our previous study, we can show an early and transient increase in both soluble isoforms of RAGE, sRAGE and esRAGE, and IL-6 immediately after trauma (Figure 1). Interestingly, these increased plasma levels cannot be observed after surgery, hinting at a major influence of a globally disturbed hemostasis as present in trauma patients compared to elective patients under general anesthesia.

3.3. Cell Surface Expression of RAGE and HLA-DR on Monocytes. In order to assess the abundance of RAGE on monocytes, we performed flow cytometry measurements. Both patients with trauma and surgical patients showed an $a b$ initio decreased abundance of RAGE on the cell surface (Figure 2(a)) as well as a trend to a general decrease of RAGE-positive monocytes compared to healthy controls (Figure 2(b)). These tendencies are maintained over all time points. In addition, as a surrogate marker of immune competence, we quantitatively measured monocytic HLA-DR surface expression. As expected, again both groups showed a significant decrease of HLA-DR expression, which persisted in the patients after trauma over the whole observation time. Despite the same overall kinetic, the expression levels of RAGE and HLA-DR in trauma patients over all time points correlate only weakly but yet significantly $(\rho=0.336, P=$ 0.007).

3.4. RAGE Ligands and Metabolic Stress after Trauma. A range of ligands from different origins and nature have been discovered for RAGE so far. We wanted to shed light on the question to which extent a panel of RAGE ligands actually occurs after trauma and might contribute to the pathophysiology. To our surprise, the plasma concentrations of the archetypical DAMP HMGB1 were changed neither immediately after trauma nor at any later time point (Figure 3(a)). In sharp contrast, S100A8 (Figure 3(b)) as well as S100A12 (Figure 3(a)) levels increased with a delayed kinetic and peaked at day 4 after trauma. The same kinetic was also found for AGE-modified proteins (Figure 4(c)). Taking a look at the other metabolic ligands, for example, CMLand MG-modified proteins, our study revealed a paradoxical kinetic with concentrations initially below healthy controls for surgical as well as trauma patients, recovering after 4 days back to normal (Figures 4(a) and 4(b)). Finally, we measured the concentration of the reactive carbonyl species methylglyoxal (MG) in the plasma, which is a relevant driver for 

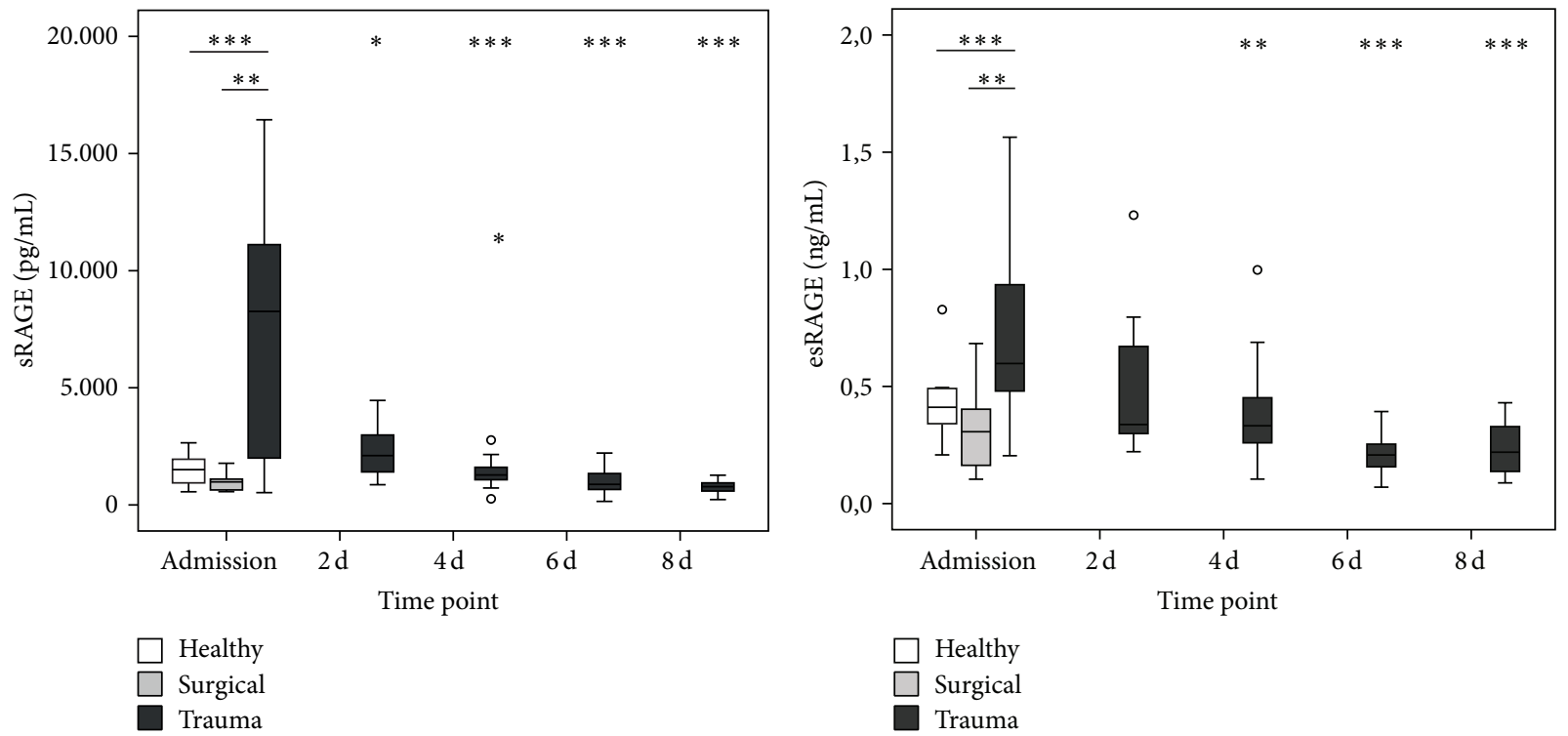

(a)

(b)

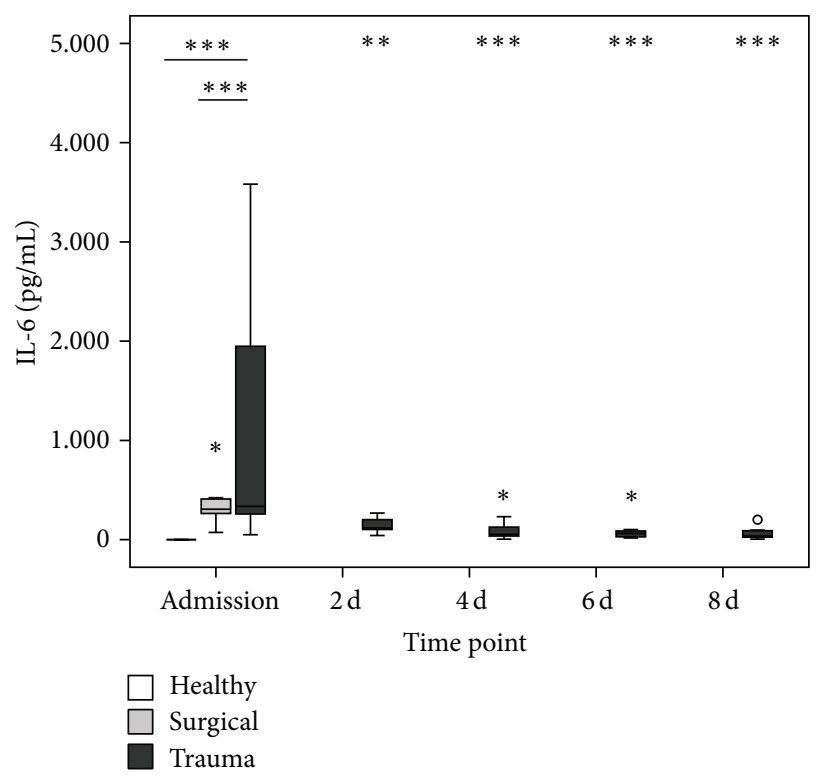

(c)

FIGURE 1: Plasma concentrations of RAGE isoforms and IL-6 after trauma. sRAGE (a), esRAGE (b), and IL-6 (c) levels at admission $(n=$ $16 / 10 / 10$ for trauma/surgical/healthy) and $2 \mathrm{~d}(n=16), 4 \mathrm{~d}(n=15), 6 \mathrm{~d}(n=11)$, and $8 \mathrm{~d}$ after trauma $(n=11) .{ }^{*} P<0.05,{ }^{* *} P<0.01$, and ${ }^{* * *} P<0.001$ compared to trauma at admission (Mann-Whitney $U$ ).

the generation of AGEs (Figure 4(c)). In contrast to the decreased concentrations of CML- and MG-modified proteins, MG actually peaked very early exclusively in trauma patients and dropped back to normal values at day 2 after trauma. Overall, the results indicate an interwoven occurrence of early (IL-6, soluble RAGE, and methylglyoxal) and late (leucocytes, S100A8 + A12, and AGE-modified proteins) stakeholders of the immune response on the basis of an impaired immune function and low abundance of RAGE on monocytes in trauma patients. Moreover, the delayed generation of AGEs might be fueled by the high availability of glucose, which is a common feature of critical care patients.

\section{Discussion}

Our study is the first to our knowledge which examines the RAGE axis in patients after severe trauma in a longitudinal approach, including metabolic ligands and drivers, soluble receptor isoforms, and monocyte surface expression. Evidence for the importance of RAGE in this setting derives amongst others from polymorphism studies, presenting an association of infectious complications after trauma with a single nucleotide polymorphism (SNP) in the promotor region regulating RAGE expression [20]. Moreover, as a consequence of high basal expression levels of RAGE in 


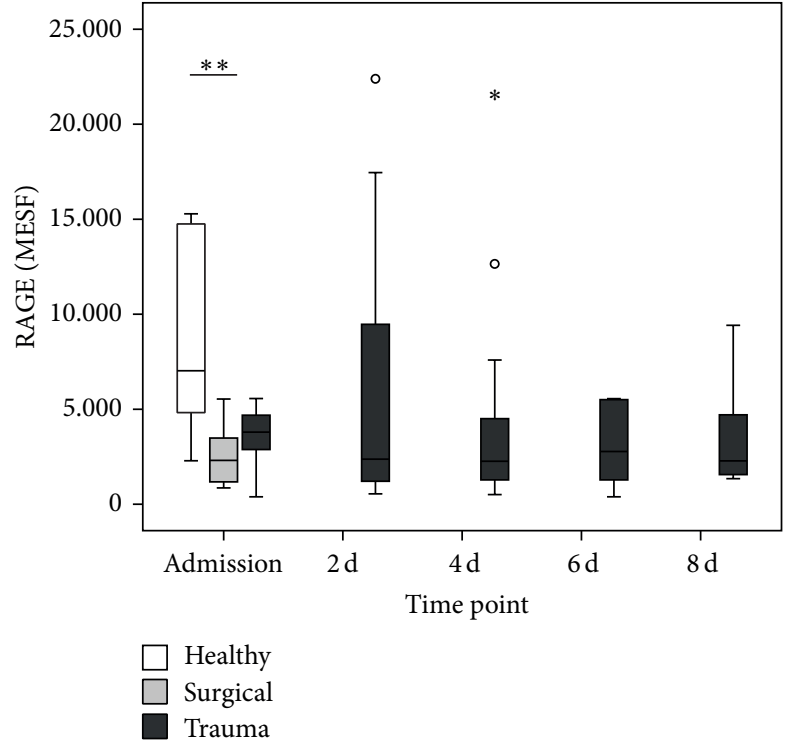

(a)

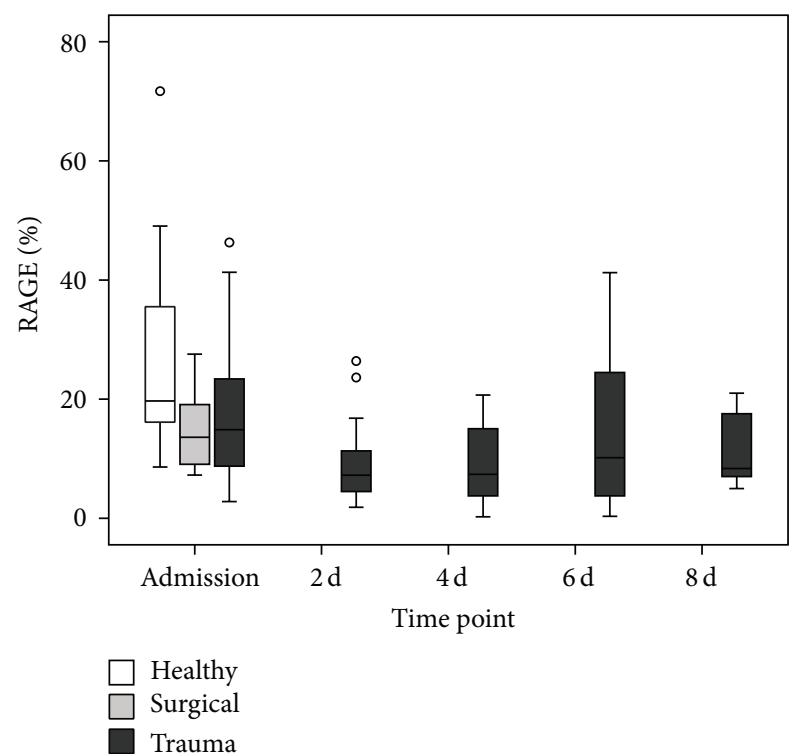

(b)

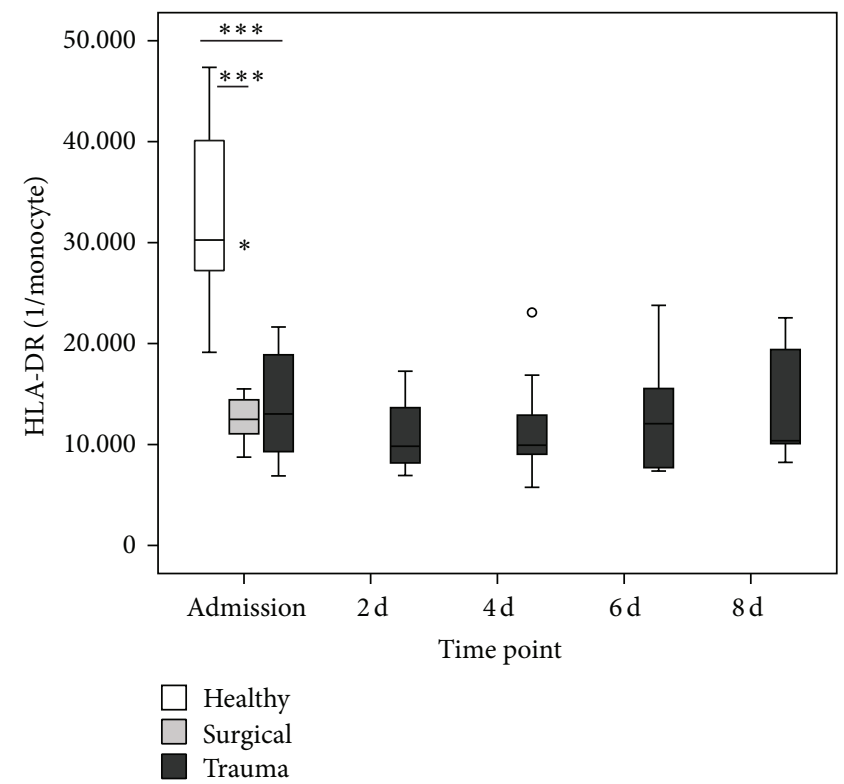

(c)

FIGURE 2: Cell surface expression of RAGE and HLA-DR. Results for RAGE are given as Molecules of Soluble Fluorochrome (MESF) (a) and percentage of $\mathrm{RAGE}^{+}$monocytes compared to isotype (b), HLA-DR as mean amount of molecules per monocyte (c) at admission ( $n=16 / 10 / 10$ for trauma/surgical/healthy) and $2 \mathrm{~d}(n=16), 4 \mathrm{~d}\left(n=14\right.$ for RAGE), $6 \mathrm{~d}(n=10)$, and $8 \mathrm{~d}$ after trauma $(n=8)$. ${ }^{*} P<0.05$, ${ }^{* *} P<0.01$, and ${ }^{* * *} P<0.001$ compared to trauma at admission (Mann-Whitney $U$ ).

the lung cells [21], ligation of the receptor might be causative to subsequent pulmonary dysfunction [22].

Earlier studies of our group and others show that soluble isoforms occur very rapidly and transiently after trauma and are associated, for example, with injury severity $[16,17]$. These results are in line with the findings of our current study and are extended here in terms of control groups. In fact, patients after surgery do not show the drastic kinetic of RAGE occurrence like trauma patients, hinting at a molecular mechanism of generation which is rather depending on a deregulated homeostasis compared to the actual tissue injury, which is to the same extent present in the patients after major abdominal surgery. This idea is further supported by the fact that also patients with sepsis, a condition that represents the infectious counterpart to the sterile situation after trauma, exhibit high plasmatic levels of sRAGE [13, 14]. In the present study, plasma levels of IL- 6 also showed a remarkable difference between patients after surgery and trauma, proving an early and fulminant SIRS as stated some decades ago [23]. Despite the differences in response between the groups, DAMPs have been proven to be important mediators of immune reactions after trauma and other conditions [6]. 


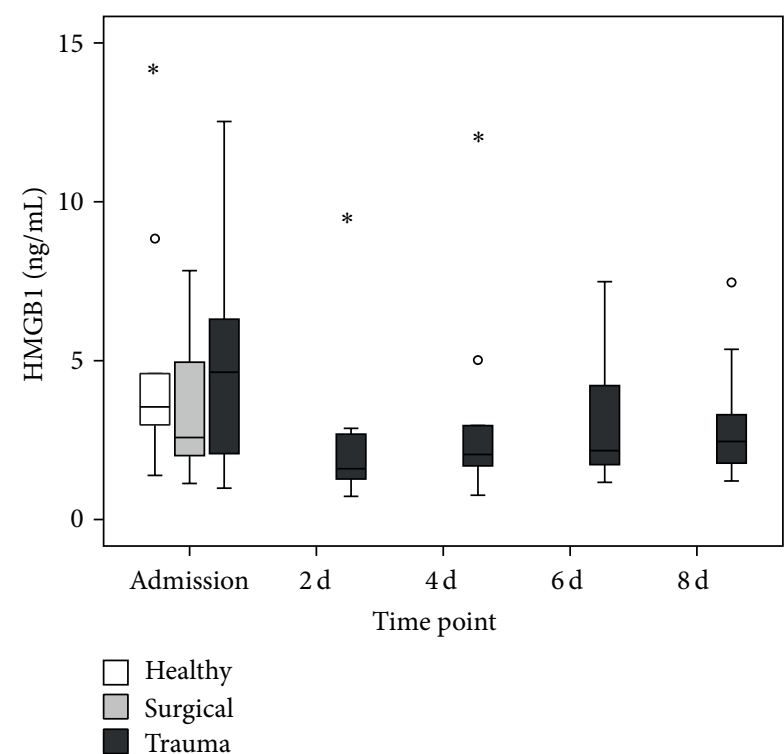

(a)

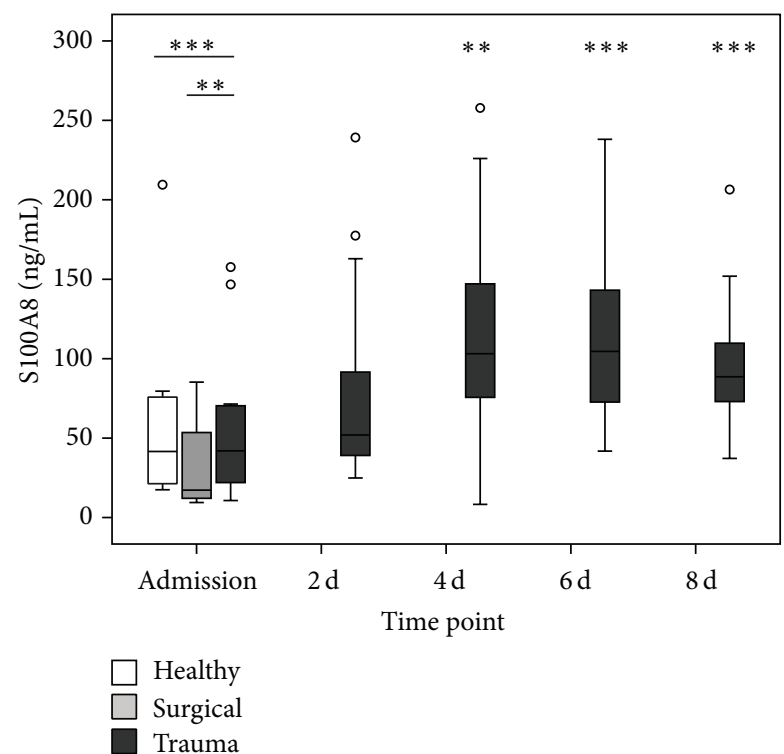

(b)

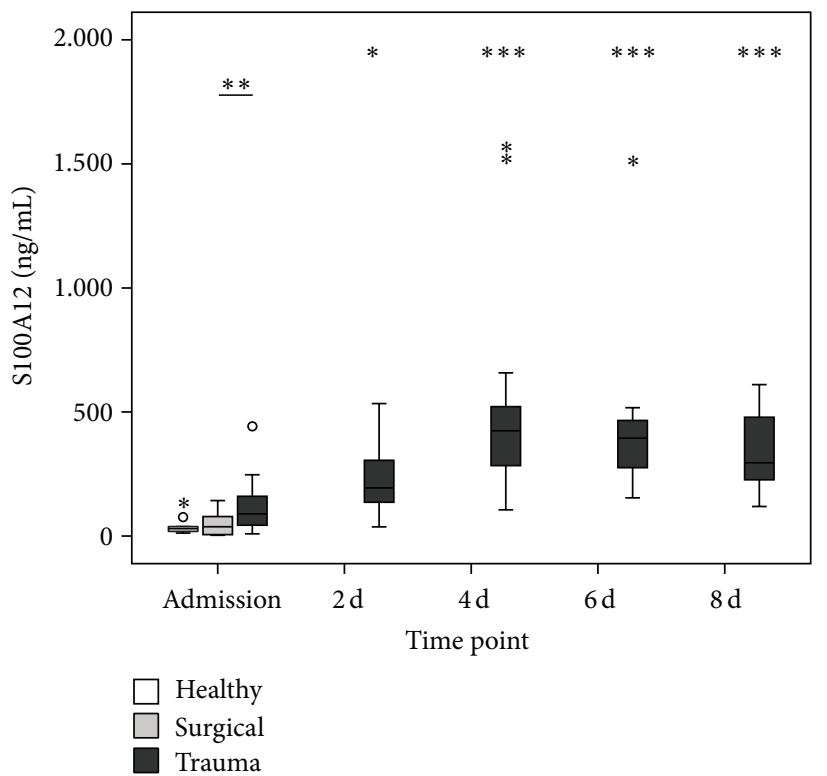

(c)

FIGURE 3: Plasma concentrations of proteinergic RAGE ligands after trauma. HMGB1 (a), S100A8 (b), and S100A12 (c) levels at admission ( $n=16 / 10 / 10$ for trauma/surgical/healthy) and $2 \mathrm{~d}(n=16), 4 \mathrm{~d}(n=15), 6 \mathrm{~d}(n=11)$, and $8 \mathrm{~d}$ after trauma $(n=11) .{ }^{*} P<0.05,{ }^{* *} P<0.01$, and ${ }^{* * *} P<0.001$ compared to trauma at admission (Mann-Whitney $U$ ).

For HMGB1, an archetypical DAMP with high nuclear abundance in all somatic cells, the release after trauma has been reported earlier by Cohen and coworkers [24]. This is however conflicting with the results of our present study, where we cannot see any clear changes in HMGB1 levels. Nevertheless, HMGB1 has been reported earlier to induce a cross-tolerance to LPS [25] as well as a secondary state of "functional exhaustion" in mature monocytes [26]. Besides HMGB1, also a large arsenal of other DAMPs have been reported to be immunogenic drivers, not least our own mitochondrial DNA [27].

To estimate whether high levels of soluble RAGE are associated with a decreased surface expression on monocytes, flow cytometry experiments were performed. After an initial drop, the abundance of RAGE tended to increase in a portion of patients and then dropped again, while the proportion of $\mathrm{RAGE}^{+}$monocytes stayed more or less the same. Although there was no significant difference, we cannot rule out that monocytes lose a part of their RAGE repertoire through, for example, enzymatic shedding by the metalloproteinase ADAM10 [28], what might be a counterregulatory mechanism to avoid excessive activation of inflammatory signaling cascades. In support of this idea, our patients show a persistent decrease of HLA-DR over the whole observation time, a marker that is a valid surrogate for monocytic immune function [29]. Nevertheless, a residual expression of RAGE on 


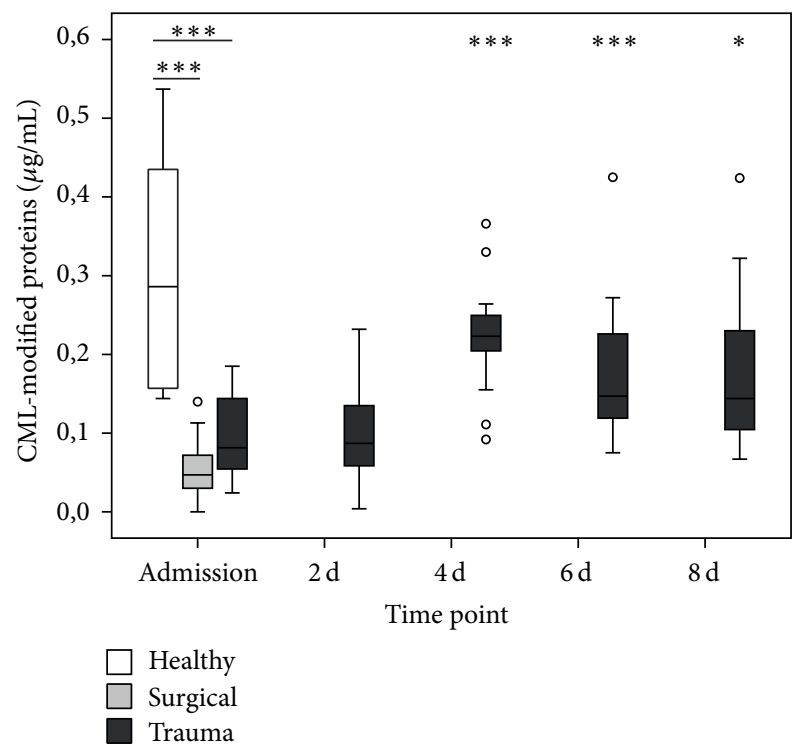

(a)

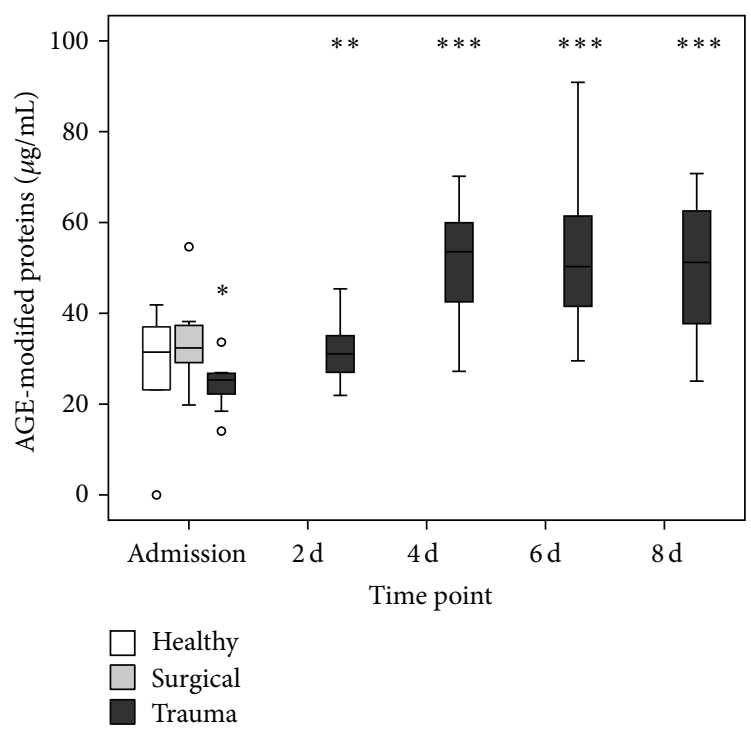

(c)

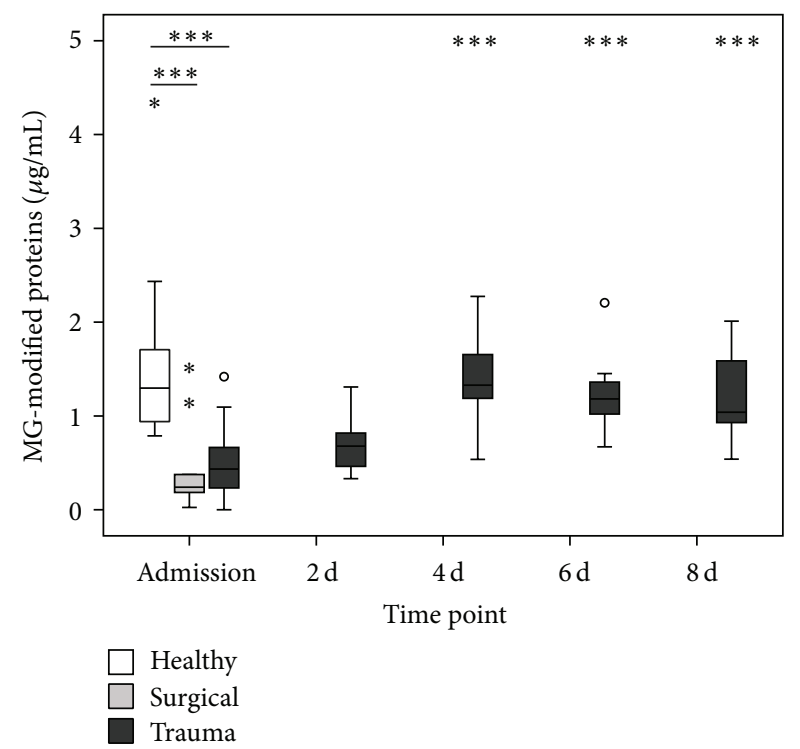

(b)

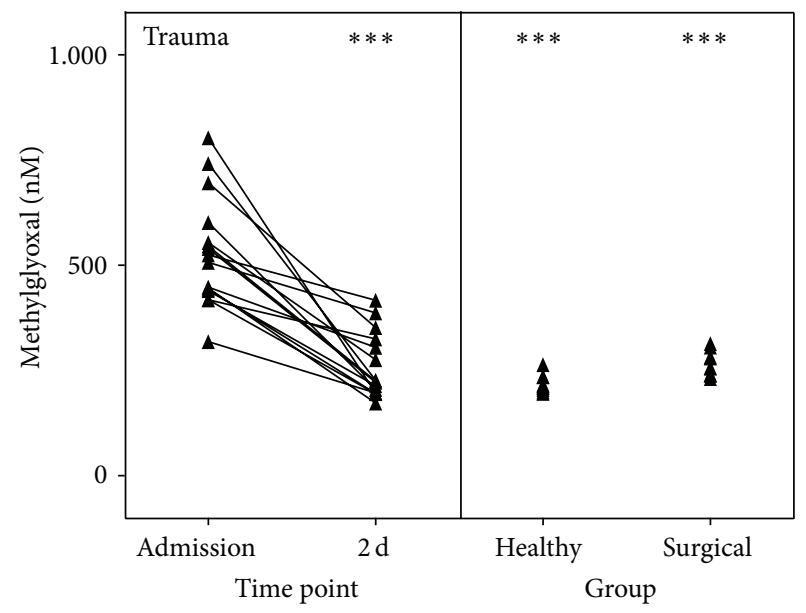

(d)

FIGURE 4: Plasma concentrations of metabolic RAGE ligands and methylglyoxal after trauma. CML- (a), MG- (b), and AGE-modified protein (c) and methylglyoxal (d) levels at admission $(n=16 / 10 / 10$ for trauma/surgical/healthy) and $2 \mathrm{~d}(n=16), 4 \mathrm{~d}(n=15), 6 \mathrm{~d}(n=11)$, and $8 \mathrm{~d}$ after trauma $(n=11) .{ }^{*} P<0.05,{ }^{* *} P<0.01$, and ${ }^{* * *} P<0.001$ compared to trauma at admission (Mann-Whitney $U$ ).

monocytes might still be sufficient for a basal signaling level, fueled by the delayed occurrence of immunogenic ligands of RAGE, namely, S100A8 and S100A12. The last one has also been reported to be capable of binding to and activating TLR4 in a proinflammatory fashion [30]. The same holds also true for S100A8, which has above all proven to induce a secondary state of hyporesponsiveness in monocytes [31], mimicking the clinical picture we observe in our cohort and similar to the well-known state of endotoxin tolerance [32]. In addition, we also found AGE-modified proteins to be increased in a delayed but profound manner. This might be an indicator of metabolically relevant changes in trauma patients, leading primarily to increased glucose levels and secondarily, in combination with the oxidative stress, to a generation of the aforementioned AGEs. One explanation might be the known presence of a transient, inflammation-induced insulin resistance [33], inducing diabetes-like metabolic conditions. Accordingly, "real" diabetes mellitus has been shown to be associated with an increased plasmatic AGE level and interestingly also a higher plasmatic methylglyoxal (MG) load [34], a feature we also observe in our trauma patients and we found out earlier in patients with sepsis [35]. MG is a member of reactive carbonyl species, which might influence cellular function in several ways. In the context of diabetes, MG has been shown to very distinctively modify and hyperactivate $\mathrm{Na}_{\mathrm{v}} 1.8$, a sodium ion channel expressed on nociceptive sensory fibers, resulting in the sensation of neuropathic pain [36]. Interestingly, $\mathrm{Na}_{\mathrm{v}} 1.8$ can also be activated by 
bacteria-derived formylated peptides, leading to pain but even more astonishingly to a modulation of the local immune reaction [37]. Combining these findings with our own results of high MG levels after trauma, two assumptions arise: first, there seems to be an intracellular catabolic change, leading to shunting of the energy flux from glycolysis to MG generation rather than to citrate cycle. Secondly, MG generation might be at the same time a proinflammatory stimulus as well as a trigger for an early mechanism to counteract excessive inflammation and thereby prevent harm to the organism.

Our pilot study implies some limitations regarding small sample size as well as the singular sampling of the surgical patients. In addition, trauma patients discharged from intensive or intermediate care unit were not followed up, what leads to a "survivorship bias."

In summary, our study sheds light on all levels of the RAGE axis, from ligands over decoy receptors to surface RAGE expression. Intriguingly, we found a late occurrence of the proteinergic ligands S100A8 and S100A12 as well as high levels of AGE-modified proteins. In combination with the profound depression of immune responsiveness, which renders the patients vulnerable for an infectious encounter and the lack of IL- 6 on later time points, one might hypothesize RAGE (and its ligands) to be contributing drivers to this refractory immune condition. As common in the immune system, the role of RAGE might be bivalent one, initially driving the sterile systemic immune reaction together with other receptors known to recognize DAMPs (e.g., TLR4) and secondarily a responsibility for the compensatory immune dysfunction of phagocytes (e.g., monocytes). However, as our study is based on the clinical setting, we are not able to unravel causal relationships. This task must be performed in a controlled setting, for example, cell culture in order to accurately pinpoint the role of RAGE in cellular function. If our very early and preliminary findings would hold true, one might speculate in trauma patients with sustained immune dysfunction about the rationale for a "paradoxical" treatment of disrupting the RAGE, ligand interaction by, for example, small molecule inhibitors (e.g., FPS-ZM1), which are currently tested in clinical trials for their potential use to prevent RAGE-mediated amyloid transport over the blood brain barrier [38]. The ultimate goal must be to balance out immunity in our patients to have as much response as needed, but without losing responsiveness in a compensatory fashion.

\section{Conflict of Interests}

The authors declare that there is no conflict of interests regarding the publication of this paper.

\section{Authors' Contribution}

Florian Uhle, Christoph Lichtenstern, Markus Alexander Weigand, and Katja Weismüller conducted design and organization of the study and performed sample measurements, data analysis and collection, interpretation of the results, and writing of the paper. Thorsten Brenner, Christian Koch, Christian Heiss, and Stefan Hofer conducted data analysis and collection, interpretation of the results, and writing of the paper. Thomas Fleming and Peter Paul Nawroth performed sample measurements, interpretation of results, and critical revision of the paper.

\section{Acknowledgments}

This study was supported by an internal research grant of the University Hospital Giessen to Katja Weismüller and Christoph Lichtenstern. Peter Paul Nawroth and Thomas Fleming received financial support from the Collaborative Research Centre 1118 (DFG).

\section{References}

[1] C. J. L. Murray and A. D. Lopez, "Alternative projections of mortality and disability by cause 1990-2020: global Burden of Disease study," The Lancet, vol. 349, no. 9064, pp. 1498-1504, 1997.

[2] E. G. Krug, G. K. Sharma, and R. Lozano, "The global burden of injuries," American Journal of Public Health, vol. 90, no. 4, pp. 523-526, 2000.

[3] H. E. Campbell, E. A. Stokes, D. N. Bargo et al., "Quantifying the healthcare costs of treating severely bleeding major trauma patients: a national study for England," Critical Care, vol. 19, article 276, 2015.

[4] M. Keel and O. Trentz, "Pathophysiology of polytrauma," Injury, vol. 36, no. 6, pp. 691-709, 2005.

[5] M. Levi and T. van der Poll, "Inflammation and coagulation," Critical Care Medicine, vol. 38, no. 2, supplement, pp. S26-S34, 2010.

[6] M. E. Bianchi, "DAMPs, PAMPs and alarmins: all we need to know about danger," Journal of Leukocyte Biology, vol. 81, no. 1, pp. 1-5, 2007.

[7] T. Kislinger, C. Fu, B. Huber et al., " $N^{\varepsilon}$-(carboxymethyl)lysine adducts of proteins are ligands for receptor for advanced glycation end products that activate cell signaling pathways and modulate gene expression," The Journal of Biological Chemistry, vol. 274, no. 44, pp. 31740-31749, 1999.

[8] J. Xue, V. Rai, D. Singer et al., "Advanced glycation end product recognition by the receptor for AGEs," Structure, vol. 19, no. 5, pp. 722-732, 2011.

[9] Y. K. Chuah, R. Basir, H. Talib, T. H. Tie, and N. Nordin, "Receptor for advanced glycation end products and its involvement in inflammatory diseases," International Journal of Inflammation, vol. 2013, Article ID 403460, 15 pages, 2013.

[10] A. Goldin, J. A. Beckman, A. M. Schmidt, and M. A. Creager, "Advanced glycation end products: sparking the development of diabetic vascular injury," Circulation, vol. 114, no. 6, pp. 597605, 2006.

[11] V. P. Singh, A. Bali, N. Singh, and A. S. Jaggi, "Advanced glycation end products and diabetic complications," Korean Journal of Physiology and Pharmacology, vol. 18, no. 1, pp. 1-14, 2014.

[12] S. F. Yan, R. Ramasamy, and A. M. Schmidt, "Soluble RAGE: therapy and biomarker in unraveling the RAGE axis in chronic disease and aging," Biochemical Pharmacology, vol. 79, no. 10, pp. 1379-1386, 2010.

[13] C. Bopp, S. Hofer, J. Weitz et al., "sRAGE is elevated in septic patients and associated with patients outcome," Journal of Surgical Research, vol. 147, no. 1, pp. 79-83, 2008. 
[14] H. Matsumoto, N. Matsumoto, H. Ogura et al., "The clinical significance of circulating soluble RAGE in patients with severe sepsis," Journal of Trauma and Acute Care Surgery, vol. 78, no. 6, pp. 1086-1094, 2015.

[15] F. Kimura, H. Shimizu, H. Yoshidome, M. Ohtsuka, and M. Miyazaki, "Immunosuppression following surgical and traumatic injury," Surgery Today, vol. 40, no. 9, pp. 793-808, 2010.

[16] F. Uhle, D. van den Nouland, S. Little et al., "Plasmatic isoforms of cytokeratin 18 and RAGE after severe trauma: a longitudinal cohort study," Journal of Trauma and Acute Care Surgery, vol. 77, no. 4, pp. 577-584, 2014.

[17] M. J. Cohen, M. Carles, K. Brohi et al., "Early release of soluble receptor for advanced glycation endproducts after severe trauma in humans," Journal of Trauma-Injury, Infection \& Critical Care, vol. 68, no. 6, pp. 1273-1278, 2010.

[18] P. J. Thornalley, A. Langborg, and H. S. Minhas, "Formation of glyoxal, methylglyoxal and 3-deoxyglucosone in the glycation of proteins by glucose," The Biochemical Journal, vol. 344, part 1, pp. 109-116, 1999.

[19] A. C. McLellan, S. A. Phillips, and P. J. Thornalley, "The assay of methylglyoxal in biological systems byderivatization with 1,2diamino-4,5-dimethoxybenzene," Analytical Biochemistry, vol. 206, no. 1, pp. 17-23, 1992.

[20] L. Zeng, "Rs1800625 in the receptor for advanced glycation end products gene predisposes to sepsis and multiple organ dysfunction syndrome in patients with major trauma," Critical Care, vol. 19, article 1, 7 pages, 2015.

[21] S. T. Buckley and C. Ehrhardt, "The receptor for advanced glycation end products (RAGE) and the lung," Journal of Biomedicine and Biotechnology, vol. 2010, Article ID 917108, 11 pages, 2010.

[22] D. J. Weber, A. S. A. Gracon, M. S. Ripsch et al., "The HMGB1RAGE axis mediates traumatic brain injury-induced pulmonary dysfunction in lung transplantation," Science Translational Medicine, vol. 6, no. 252, Article ID 252ra124, 2014.

[23] C. Martin, C. Boisson, M. Haccoun, L. Thomachot, and J.-L. Mege, "Patterns of cytokine evolution (tumor necrosis factor- $\alpha$ and interleukin-6) after septic shock, hemorrhagic shock, and severe trauma," Critical Care Medicine, vol. 25, no. 11, pp. 18131819, 1997.

[24] M. J. Cohen, K. Brohi, C. S. Calfee et al., "Early release of high mobility group box nuclear protein 1 after severe trauma in humans: role of injury severity and tissue hypoperfusion," Critical Care, vol. 13, article R174, 2009.

[25] R. K. Aneja, A. Tsung, H. Sjodin et al., "Preconditioning with high mobility group box 1 (HMGB1) induces lipopolysaccharide (LPS) tolerance," Journal of Leukocyte Biology, vol. 84, no. 5, pp. 1326-1334, 2008.

[26] A. Liesz, A. Dalpke, E. Mracsko et al., "DAMP signaling is a key pathway inducing immune modulation after brain injury," The Journal of Neuroscience, vol. 35, no. 2, pp. 583-598, 2015.

[27] Q. Zhang, M. Raoof, Y. Chen et al., "Circulating mitochondrial DAMPs cause inflammatory responses to injury," Nature, vol. 464, no. 7285, pp. 104-107, 2010.

[28] A. Raucci, S. Cugusi, A. Antonelli et al., "A soluble form of the receptor for advanced glycation endproducts (RAGE) is produced by proteolytic cleavage of the membrane-bound form by the sheddase a disintegrin and metalloprotease 10 (ADAM10)," The FASEB Journal, vol. 22, no. 10, pp. 3716-3727, 2008.

[29] F. Venet, S. Tissot, A.-L. Debard et al., "Decreased monocyte human leukocyte antigen-DR expression after severe burn injury: correlation with severity and secondary septic shock," Critical Care Medicine, vol. 35, no. 8, pp. 1910-1917, 2007.

[30] D. Foell, H. Wittkowski, C. Kessel et al., "Proinflammatory S100A12 can activate human monocytes via toll-like receptor 4," American Journal of Respiratory and Critical Care Medicine, vol. 187, no. 12, pp. 1324-1334, 2013.

[31] J. Austermann, J. Friesenhagen, S. Fassl et al., "Alarmins MRP8 and MRP14 induce stress tolerance in phagocytes under sterile inflammatory conditions," Cell Reports, vol. 9, no. 6, pp. 21122124, 2014.

[32] E. López-Collazo and C. del Fresno, "Pathophysiology of endotoxin tolerance: mechanisms and clinical consequences," Critical Care, vol. 17, no. 6, article 242, 2013.

[33] S. E. Shoelson, J. Lee, and A. B. Goldfine, "Inflammation and insulin resistance," The Journal of Clinical Investigation, vol. 116, no. 7, pp. 1793-1801, 2006.

[34] N. Ahmed, R. Babaei-Jadidi, S. K. Howell, P. J. Beisswenger, and P. J. Thornalley, "Degradation products of proteins damaged by glycation, oxidation and nitration in clinical type 1 diabetes," Diabetologia, vol. 48, no. 8, pp. 1590-1603, 2005.

[35] T. Brenner, T. Fleming, F. Uhle et al., "Methylglyoxal as a new biomarker in patients with septic shock: an observational clinical study," Critical Care, vol. 18, article 683, 2014.

[36] A. Bierhaus, T. Fleming, S. Stoyanov et al., "Methylglyoxal modification of Nav 1.8 facilitates nociceptive neuron firing and causes hyperalgesia in diabetic neuropathy," Nature Medicine, vol. 18, no. 6, pp. 926-933, 2012.

[37] I. M. Chiu, B. A. Heesters, N. Ghasemlou et al., "Bacteria activate sensory neurons that modulate pain and inflammation," Nature, vol. 501, no. 7465, pp. 52-57, 2013.

[38] R. Deane, I. Singh, A. P. Sagare et al., "A multimodal RAGEspecific inhibitor reduces amyloid $\beta$-mediated brain disorder in a mouse model of Alzheimer disease," The Journal of Clinical Investigation, vol. 122, no. 4, pp. 1377-1392, 2012. 


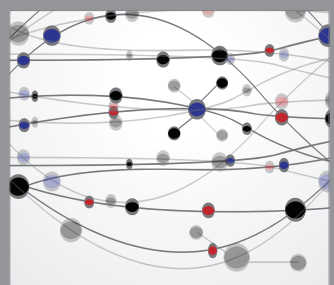

The Scientific World Journal
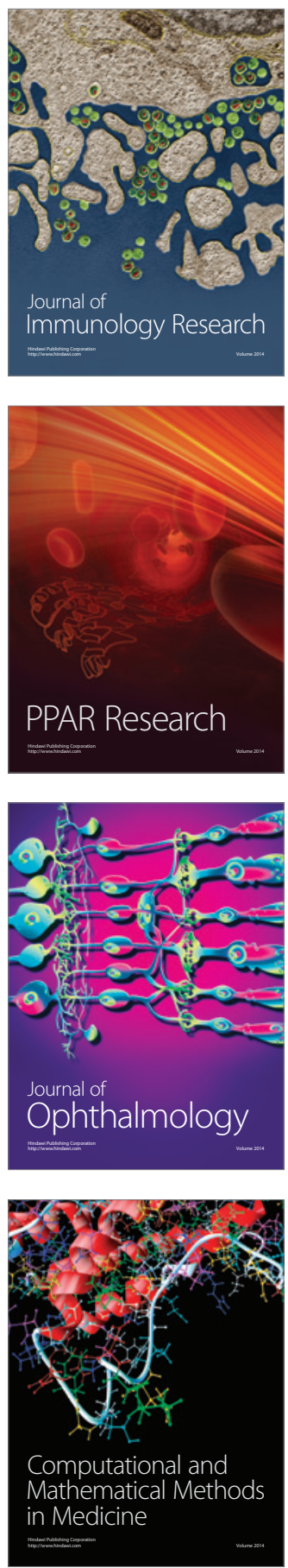

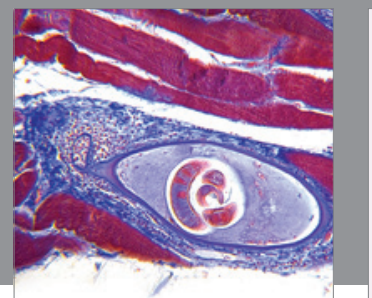

Gastroenterology

Research and Practice
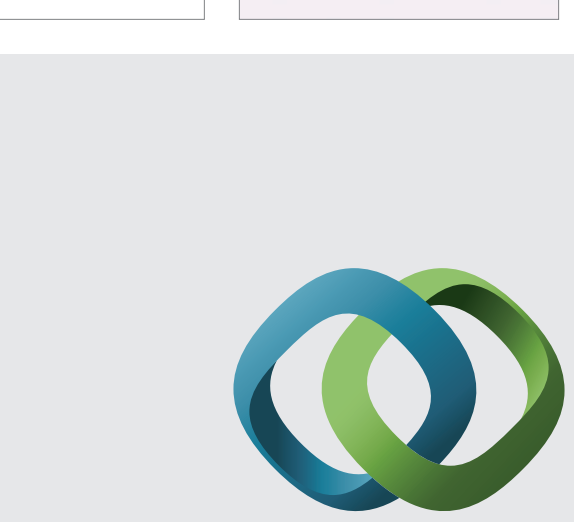

\section{Hindawi}

Submit your manuscripts at

http://www.hindawi.com
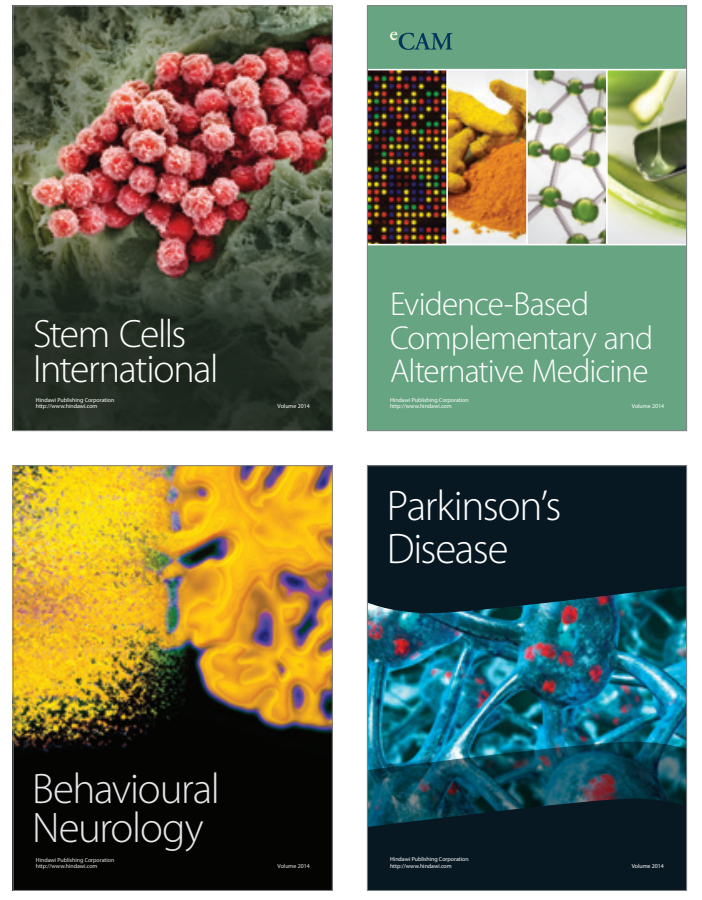
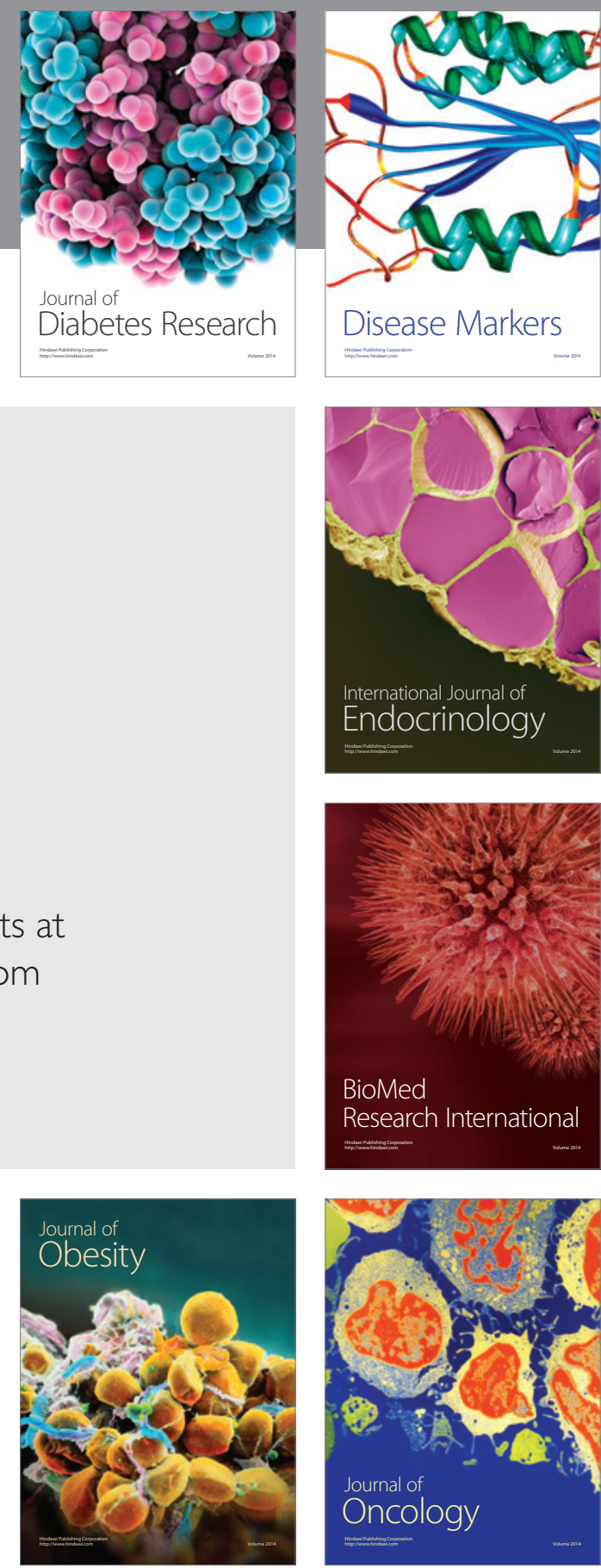

Disease Markers
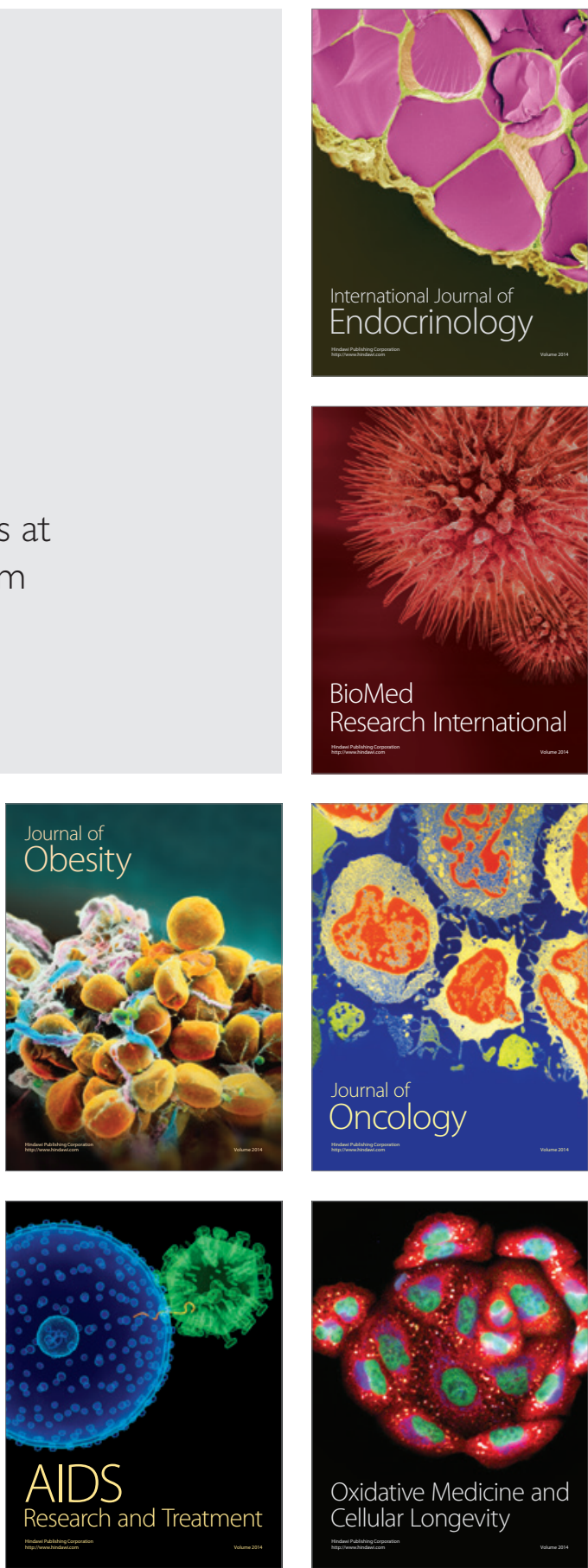